\title{
Diffuse-Reflectance Spectroscopy Using a Frequency-Switchable Terahertz Quantum Cascade Laser
}

\author{
Alexander Valavanis, Siddhant Chowdhury, Andrew D. Burnett, Adam R. Clarkson, David R. Bacon, \\ Suraj P. Khanna, Alexander Giles Davies, Edmund H. Linfield, and Paul Dean
}

\begin{abstract}
We demonstrate diffuse-reflectance (DR) spectroscopy of powders using a discretely-tunable terahertz-frequency quantum cascade laser (THz QCL) with a heterogeneous active region. DR signatures were obtained at frequencies of 3.06, $3.21,3.28$, and $3.35 \mathrm{THz}$, and the relative absorption coefficients were inferred at each frequency using a Kubelka-Munk (KM) scattering model. The spectral lineshapes reproduce the absolute Beer-Lambert (BL) absorption spectra of a range of materials, which were also measured using conventional transmission-mode THz time-domain spectroscopy. It is shown that the DR technique works reliably for materials that include pharmaceutical compounds and foodstuffs, with $B L$ absorption coefficients in the range $2-10 \mathrm{~mm}^{-1}$. This method is potentially suitable for automated material identification, without any requirement for $a$ priori knowledge of the refractive index or scattering properties of the sampled material.
\end{abstract}

Index Terms-Infrared spectra, quantum cascade lasers, spectroscopy, submillimeter-wave technology.

\section{INTRODUCTION}

$\mathbf{N}$ UMEROUS potential applications exist for terahertz-frequency $(\mathrm{THz})$ spectroscopy and imaging, including the characterization of pharmaceutical [1] and biomedical samples [2], and the detection of illicit drugs [3] and explosives [4]. In this context, reflectance-measurement techniques often present practical advantages over transmittance geometries, owing

Manuscript received June 12, 2015; revised October 15, 2015; accepted December 23, 2015. Date of publication February 08, 2016; date of current version March 21, 2016. This work was supported by the Engineering and Physical Sciences Research Council (EPSRC), U.K. under Grant EP/J002356/1 and Grant EP/J017671/1, by the ERC under Grant 247375 ("TOSCA") and Grant 228035 ("NOTES"), and by a number of U.K. government departments and agencies under the CONTEST Innovative Research Call in Explosives and Weapons Detection 2007. (Corresponding author: Alexander Valavanis.)

A. Valavanis, S. Chowdhury, D. R. Bacon, A. G. Davies, E. H. Linfield, and P Dean are with the Institute of Microwaves and Photonics, School of Electronic and Electrical Engineering, University of Leeds, Leeds, LS2 9JT, U.K. (e-mail: a.valavanis@leeds.ac.uk).

A. D. Burnett is with the Institute of Microwaves and Photonics, School of Electronic and Electrical Engineering, University of Leeds, Leeds, LS2 9JT, U.K., and also with the School of Chemistry, University of Leeds, Leeds, LS2 9JT, U.K

A. R. Clarkson was with the Institute of Microwaves and Photonics, School of Electronic and Electrical Engineering, University of Leeds, Leeds, LS2 9JT, U.K. He is now with ARM Ltd., Sheffield, S1 4EB, U.K..

S. P. Khanna was with the Institute of Microwaves and Photonics, School of Electronic and Electrical Engineering, University of Leeds, Leeds, LS2 9JT, U.K. He is now with the National Physical Laboratory, New Delhi, 110012, India..

Color versions of one or more of the figures in this paper are available online at http://ieeexplore.ieee.org.

Digital Object Identifier 10.1109/TTHZ.2016.2515082 to the ability to study thick or highly-absorbing samples, as well as their potential for stand-off interrogation of targets [5]. Diffuse-reflectance (DR) techniques, which sense the radiation scattered from rough surfaces or powders, are dependent upon both the scattering cross-section and the bulk-absorption properties of media [6], and can hence reveal more detailed information about samples than specular-reflection techniques. Furthermore, smooth $\mathrm{THz}$-transparent packaging materials do not introduce DR artifacts [7], and diffuse reflections may be detected over a large solid-angle, thus simplifying the alignment of detection optics. The latter is advantageous in security-screening of hazardous explosive samples, in which large stand-off distances (through air) are required [5].

$\mathrm{THz}$ quantum cascade lasers ( $\mathrm{THz}$ QCLs) are attractive sources for DR spectroscopy, owing to their high emission power and narrow linewidth [7], and they have previously been used to determine the $\mathrm{THz}$ absorption (at a single frequency) of a range of powdered materials [8]. To date, however, multi-frequency DR spectroscopy with THz QCLs has not been achieved, owing to their limited tunability. In this work, we use a frequency-switchable $\mathrm{THz}$ QCL, based on a heterogeneous resonant-phonon-depopulation active region design [9], as the radiation source in a DR spectroscopy system. DR measurements of powdered materials are obtained at frequencies of 3.06, 3.21, 3.28, and 3.35 THz. The DR of a cellulose powder sample is shown to decrease monotonically with respect to the concentration of the material in an admixture with THz-transparent polytetrafluoroethylene (PTFE). A Kubelka-Munk (KM) model is then used to determine the relative absorption coefficient of a wide range of materials, and the results are shown to reproduce the lineshape of the Beer-Lambert (BL) absorption spectra, which were obtained using $\mathrm{THz}$ time-domain spectra. The absorption coeffients, obtained from the $\mathrm{KM}$ model, are shown to be proportional to the BL absorption coefficients over a $2-10 \mathrm{~mm}^{-1}$ range, and over a wide range of powder concentrations, using a frequency-independent scaling factor.

\section{SySTEM CONFIGURATION}

The optical and electronic configuration of the multi-frequency DR system used in this work is illustrated schematically in Fig. 1, and is based on a previous single-frequency system, which we have described in detail elsewhere [7], [8]. The radiation source in the modified (multi-frequency) system was a 


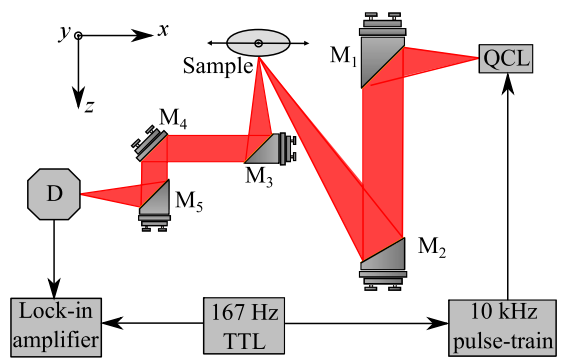

Fig. 1. Schematic illustration of the optical and electronic configuration of the DR system. A helium-cooled silicon bolometer (label 'D') was used as the detector.

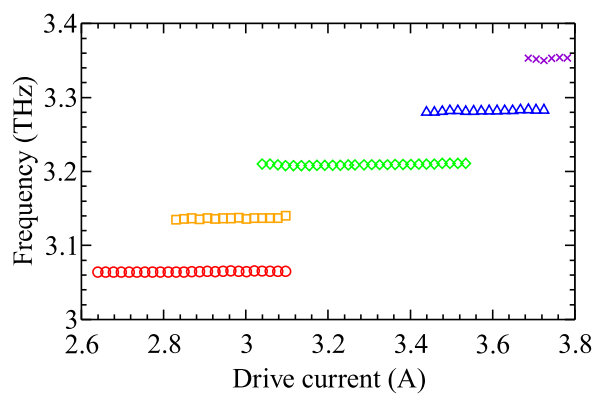

Fig. 2. Frequency of emission lines for the heterogeneous QCL at a range of drive currents, constructed from normalised FTIR spectra. Weak emission lines (spectral intensities $>20 \mathrm{~dB}$ below the principal lines) are omitted.

$\mathrm{THz}$ QCL with a heterogeneous gain medium, as previously reported in [9]. This QCL was based on a 3-well resonant-phonon design [10], and was grown using molecular-beam epitaxy with a stepped gallium effusion rate, such that the gain medium was separated into 23 modules, each with a unique emission frequency and alignment bias. The laser was processed into a $\sim 1.5$-mm-long, 140 - $\mu \mathrm{m}$-wide single-metal ridge waveguide [11], and was mounted on the cold-finger of a closed-cycle pulse-tube helium cryostat which operated at a temperature of $6 \mathrm{~K}$. A $10-\mathrm{kHz}$ current pulse train was supplied to the QCL, with $2 \%$ duty-cycle, using an Agilent $8114 \mathrm{~A}$ pulse-generator and a current-doubling transformer. These driving pulses were modulated electrically by a $167-\mathrm{Hz}$ square-wave envelope to match the peak response of the bolometric detector.

The frequencies of the principal emission peaks from the QCL were determined at a range of operating biases using Fourier-transform infrared (FTIR) spectrometry and are plotted in Fig. 2. The emission frequency is shown to undergo a series of discrete blue-shifts as the drive current is increased, giving a total emission bandwidth from $3.06-3.35 \mathrm{THz}$ with quasi-single-mode emissions at 3.06, 3.21, 3.28, and $3.35 \mathrm{THz}$. The $3.13 \mathrm{THz}$ emission line was not used in this work, as it occurs only as part of a low-intensity doublet, and this would add significant complexity to the data analysis. It should be noted that additional, higher-frequency modes may potentially be obtained at drive-currents greater than those used in this work, and the free spectral range (i.e., the separation between the modes) may be adjusted by selecting an appropriate ridge length [11]. A maximum operating temperature of $\sim 70 \mathrm{~K}$, a threshold current density of $1150 \mathrm{~A} \cdot \mathrm{cm}^{-2}$, and a peak optical power output of $\sim 8 \mathrm{~mW}$ (at an operating temperature of $5 \mathrm{~K}$ ) were determined for this device.
Radiation from the laser was collimated using a 3-in-diameter, $90^{\circ}, f / 1.7$ off-axis parabolic reflector $\left(M_{1}\right.$ in Fig. 1$)$ and focused onto the object plane at an angle-of-incidence of $30^{\circ}$ using an $f / 4.3$ reflector $\left(M_{2}\right)$. Diffuse reflections from the object plane were collected using a 2 -in-diameter, $90^{\circ}, f / 2$ reflector $\left(M_{3}\right)$, which was directed normally to the object plane so as to avoid coupling specular reflections into the detector. The diffuse reflections were coupled into a helium-cooled silicon-bolometer using a final off-axis parabolic reflector $\left(M_{5}\right)$, and the time-averaged radiative power was recorded using a lock-in amplifier, which was referenced to the $167-\mathrm{Hz}$ electrical-modulation envelope.

A set of QCL drive currents was selected to coincide with the middle of the range of operating currents for the 3.06, $3.21,3.28$ and $3.35 \mathrm{THz}$ emission modes $(2.72,3.24,3.60$, and $3.80 \mathrm{~A}$, respectively). A two-dimensional array of DR samples was acquired at each bias by scanning the samples continuously across the object plane and recording the time-averaged detector signal at $250-\mu \mathrm{m}$ intervals, using a lock-in time-constant of $10 \mathrm{~ms}$. This led to a total acquisition time of $\sim 20 \mathrm{~min}$ for a $120 \times 120$-pixel, 4-frequency DR sample array, including the time taken for motion of the mechanized translation stage. It should be noted, however, that this acquisition time could potentially be reduced by switching rapidly between the QCL drive currents within a single scan, and applying a time-division multiplexing scheme to the signal readout.

\section{SYSTEM CHARACTERIZATION}

The system was aligned using a 'knife-edge' target consisting of a rectangular section of gold-coated ISO P120 sandpaper (average particle size $=125 \mu \mathrm{m}$ ), mounted onto a smooth glass slide. The profile of the DR power was acquired as the edge of the sandpaper was translated linearly through the beam-spot in the $x$-direction (i.e., along the major axis of the focusing mirror, as indicated in Fig. 1). The optimal working distance $z$ between the sample and the focusing mirror was determined by adjusting the sample stage location, and the intensity profile was found to follow an approximate Gauss error-function with a half-width at half-maximum of $w=1.5 \mathrm{~mm}$ at the beam focus, as shown in Fig. 3(a). This was found to be independent of frequency, indicating that the system was not diffraction limited (i.e., the resolution is limited by alignment of the incident beam). This resulted in a focused beam spot-size (and wavelength) that are both larger than the powder grain sizes used in this work $(10-15 \mu \mathrm{m})$, and as such it is reasonable to treat the materials as being quasi-homogeneous scattering media in the $\mathrm{THz}$ regime. We have, however, previously demonstrated imaging resolutions of $\sim 300 \mu \mathrm{m}$ in a $2.8-\mathrm{THz}$ diffuse imaging system [7]. In principle, therefore, the present system would allow equivalent or superior resolutions after precise optical alignment, owing to the shorter emission wavelength. A second estimate of the focused beam spot size was obtained by measuring the contrast transfer functions, in the $x$ and $y$ directions, using the detector response to specular reflections from a gold-on-quartz bar resolution target.

A first-order Coltman transform [12] was applied to the data to estimate the modulation transfer function (MTF), i.e., the response to a sinusoidally varying target reflectivity. Fig. 3(b) shows that the inferred MTF remains approximately 

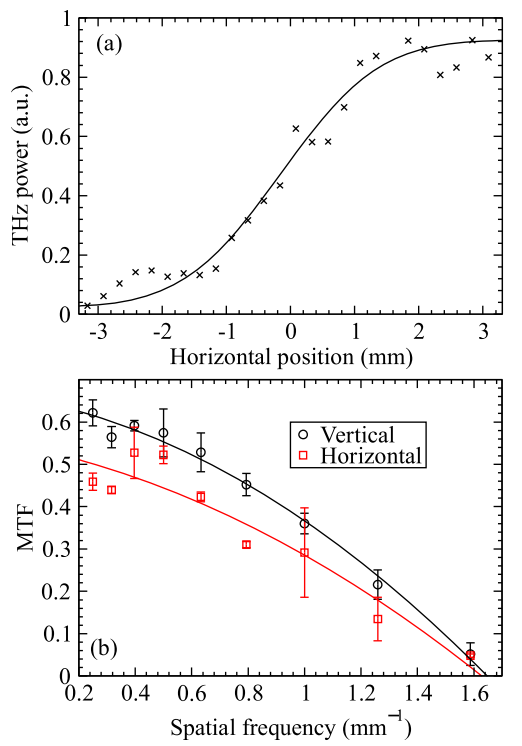

Fig. 3. (a) DR signal at $3.28 \mathrm{THz}$ acquired from a horizontal profile over a "knife-edge" target consisting of gold-coated P120 sandpaper on a glass slide. The solid line shows a regression to a Gauss error-function. (b) The modulationtransfer function (MTF) obtained from scans in the horizontal $(x)$ and vertical $(y)$ direction across a gold-on-quartz target at $3.28 \mathrm{THz}$. Error bars indicate the MTF range obtained from three independent measurements.

TABLE I

MAXIMUM SNR (DB) AT EACH EMISSION FREQUENCY (THz), FOR LOCK-IN TIME CONSTANTS OF $10 \mathrm{MS}$ AND $1 \mathrm{~s}$

\begin{tabular}{r||r||r}
\hline Frequency & SNR $(\mathbf{1 0} \mathbf{~ m s})$ & SNR $(\mathbf{1} \mathbf{~ s})$ \\
\hline \hline 3.06 & 20.5 & 40.9 \\
3.21 & 15.1 & 36.0 \\
3.28 & 23.1 & 42.8 \\
3.35 & 15.2 & 39.0 \\
\hline
\end{tabular}

constant up to a target spatial frequency of $\sim 0.6 \mathrm{~mm}^{-1}$, which corresponds to spatial period of $1.7 \mathrm{~mm}$, in good agreement with the 'knife-edge' measurements. A slightly higher resolution is obtained in the $y$ direction, owing to the elliptical shape of the focused beam spot from the $30^{\circ}$ focusing mirror.

The maximum signal-to-noise ratio (SNR) of the present system was estimated by recording the lock-in amplifier output obtained from the diffuse reflection from a single position on the gold-coated sandpaper target described above, which is here assumed to represent an ideal reflecting target. The SNR, taken as the ratio of the mean signal to its standard deviation is summarized in Table I and can be seen to lie in the range $15-25 \mathrm{~dB}$ for all four emission lines, when using a $10-\mathrm{ms}$ lock-in time constant. The SNR increases by $\sim 20 \mathrm{~dB}$ (at the expense of slower data acquisition) through the use of a $1 \mathrm{~s}$ lock-in time constant.

\section{Spectroscopy of Powdered SAmples}

A range of powdered crystalline and polycrystalline solids was selected for study in this work, each having a broad $\mathrm{THz}$ absorption resonance within the $3.0-3.4 \mathrm{THz}$ frequency band. Each powdered solid was desiccated at $40{ }^{\circ} \mathrm{C}$ for 48 hours and then ground with a pestle and mortar for 5 min to break up conglomerations, and packed loosely into a 10-ml flat-sided poly-

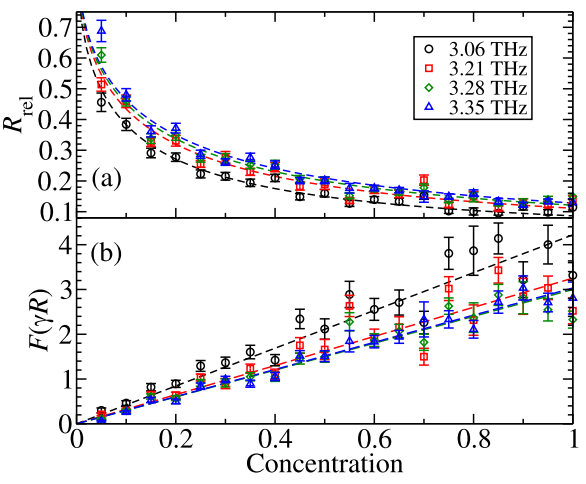

Fig. 4. (a) Diffuse reflectance of dilute samples of microgranular cellulose, relative to that of a PTFE reference powder. Results are shown at each of the four $\mathrm{THz}$ frequencies as a function of sample concentration in an admixture with PTFE. Error bars represent the absolute standard-uncertainty in the mean reflectance, and dashed lines show a regression to KM theory, using a scaling factor $\gamma=1.03$. (b) KM remission function for the same samples. Dashed lines show a regression to a linear function of concentration, as expected from BL theory.

styrene cuvette. Optical microscopy showed that the size-distribution of particles in each sample followed a Weibull probability distribution function (as expected for a disperse powder [13]), with median particle sizes ranging from $10-15 \mu \mathrm{m}$. A polytetrafluoroethylene (PTFE) sample was also prepared, as a reference material, owing to its low absorption coefficient at $\mathrm{THz}$ frequencies.

The DR from powders is intrinsically highly variable with respect to position, and this inhomogeneity gives rise to a large standard deviation in the measured diffuse reflectance. Therefore, a measurement of the mean diffuse reflectance must be taken, using a large number of independent samples across the entire powder surface. The ratio of the spatially-averaged power reflected from each sample to that of the PTFE reference was measured in order to obtain the relative reflectance, $R_{\text {rel }}=P_{\text {sample }} / P_{\text {ref }}$. In each case, measurements were acquired using a $A_{\mathrm{px}}=0.2 \times 0.2 \mathrm{~mm}^{2}$ pixel size, yielding $\sim 1000$ sampling points for each powder.

Since the standard deviation of the reflected power is merely a measure of the inhomogeneity of the powders themselves, it is not a particularly useful measure of the quality of the DR measurement. Instead, the standard uncertainty in the mean value of the DR measurements is used, which accounts for the number of independent samples taken. In our system, the pixel size was smaller than the THz beam spot size. As such, the number of independent sampling points was estimated using the area of the sampled region of the powder divided by the area of the focused beam, $N=2 N_{\mathrm{px}} A_{\mathrm{px}} / \pi w^{2}$, where $N_{\mathrm{px}}$ is the number of pixels acquired. For a typical powder sample, this yielded $N \sim 20$, depending on the exact volume of material contained within the cuvette. The standard relative uncertainty in the power is then given by $u_{P}=\sigma_{P} /\left(\mu_{P} \sqrt{N}\right)$ where $\mu_{P}$ and $\sigma_{P}$ are the mean and standard deviation of the DR power measured for a given material, respectively. It follows that the standard relative uncertainty in the relative reflectance is $u_{R}=\left(u_{P, \text { sample }}^{2}+u_{P, \text { ref }}^{2}\right)^{0.5}$.

Fig. 4(a) shows the mean relative reflectance measured for a set of samples of cellulose, which were each diluted in an admixture with PTFE to concentrations (by mass) ranging from $5 \%$ to $100 \%$. As expected, the reflectance (relative to PTFE) is shown 
to converge towards unity at all four frequencies as the concentration of cellulose is reduced to zero. A degree of spectroscopic information can be obtained immediately from this data, in that the DR increases with respect to frequency for cellulose-containing powders, implying a reduction in absorption.

We have previously demonstrated that the DR properties of scattering media can be related directly to the BL coefficient using KM theory [7], or more sophisticated approaches based on the quasi-crystalline approximation (QCA) [7], [8]. The latter techniques are advantageous in that they account for the geometry and packing density of particles, and can be used to compute the bulk absorption coefficient of materials from scattering data. However, despite their advantages, multivariate QCA methods [14] are numerically complex, and convergence of the iterative solution can be unreliable, particularly when a wide range of radiative frequencies is included in the analysis. Consequently, a $\mathrm{KM}$ analysis technique was employed in the present work. Although this approach does not directly predict the bulk absorption coefficient, it is numerically simple and robust, and (under a reasonable set of assumptions) can be used to deduce the shape of features in the absorption spectrum accurately.

This analysis approximates the powder as an infinitely-thick half-space of a homogeneous scattering medium, which is characterised by a scattering coefficient $S$ and an absorption coefficient $K$, such that [15]

$$
F\left(R_{\infty}\right)=\frac{K}{S}=\frac{\left(1-R_{\infty}\right)^{2}}{2 R_{\infty}}
$$

where $F\left(R_{\infty}\right)$ is defined as the KM remission function. The diffuse reflectance $R_{\infty}$ of the powdered medium, relative to an ideal (i.e., completely diffusing and non-absorbing) reference can be related to the measured, relative reflectance by $R_{\infty}=$ $\gamma R_{\text {rel }}$, where $\gamma$ accounts for the deviation of the PTFE reference reflectance from that of an ideal reference, and also the relative difference in the angular divergence of the radiation scattered from the two powders [7]. Furthermore, the KM absorption coefficient, $K$, is assumed to vary proportionally with the BL absorption coefficient, $\alpha$. This, in turn, increases approximately linearly with respect to the mass-concentration $c$ of the absorbing material within a weakly-absorbing matrix material [15], i.e., $K(c)=\beta \alpha(c)=\beta \alpha_{0} c$, where $\beta$ is a proportionality constant and $\alpha_{0}$ is the absorption coefficient of the bulk (undiluted) material. The (as yet undetermined) proportionality factor $\beta$ is a sample specific function, which is related to the refractive index of the powder [16]. Equation (1) may now be rewritten in terms of the two unknown constants $\gamma$ and $\beta$ and the mass-concentration of the absorbing powder.

$$
F\left(\gamma R_{\mathrm{rel}}\right)=\frac{\alpha_{0} \beta c}{S}=\frac{\left(1-\gamma R_{\mathrm{rel}}\right)^{2}}{2 \gamma R_{\mathrm{rel}}} .
$$

The inverse of (2) is obtained analytically by rearranging in terms of $R_{\text {rel }}$ and taking the negative branch of the resulting quadratic equation

$$
R_{\mathrm{rel}}=\frac{1+c \frac{\alpha_{0} \beta}{S}-\sqrt{c \frac{\alpha_{0} \beta}{S}\left(2+c \frac{\alpha_{0} \beta}{S}\right)}}{\gamma} .
$$

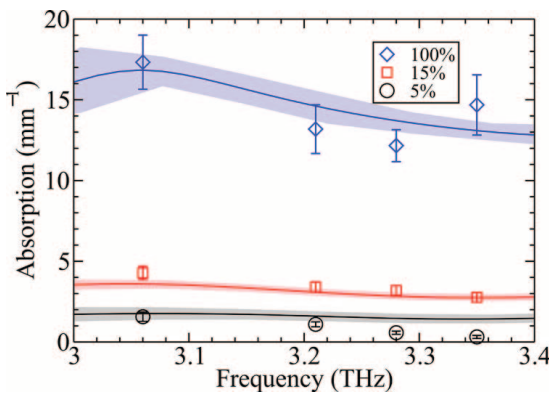

Fig. 5. Comparison between absorption coefficients of cellulose obtained using $\mathrm{THz}$ time-domain spectroscopy (solid lines) and those inferred from KM measurements using the scaling factor $S / \beta=5.23 \mathrm{~mm}^{-1}$. The standard uncertainty in absorption coefficient is represented as semi-transparent bands for the TDS data, and as error bars for the DR data.

Assuming now that the scattering coefficient is invariant with respect to concentration and frequency, the value of $\gamma$ can be found by using a linear regression to the logarithmic form

$$
\ln \left[F\left(\gamma R_{\mathrm{rel}}\right)\right]=\ln \left(\frac{\alpha_{0} \beta}{S}\right)+\ln (c)
$$

such that the gradient of a plot of $\ln \left[F\left(\gamma R_{\text {rel }}\right)\right]$ against $\ln (c)$ has a gradient of 1 and intercept, $\ln \left(\alpha_{0} \beta / S\right)$. Frequency-independent values of $\gamma=1.03$ and $\ln \left[\alpha_{0} \beta / S\right]=1.22$ were found for the cellulose samples in Fig. 4(a). The resulting functional form from (3) is plotted as dashed curves in the figure, and is shown to provide an excellent fit to the experimental data. The corresponding $\mathrm{KM}$ remission function is plotted for each of the cellulose samples in Fig. 4(b) using the same fitting parameters. The linearity of the plots is confirmed for all frequencies (with a Theil uncertainty coefficient, $U<0.2$ in all cases), as expected according to (2), thus validating our analytical model. In this figure, the standard uncertainty in the KM remission function has been computed according to the expression

$$
F\left(\gamma R_{\mathrm{rel}}\right) u_{F} \approx \gamma R_{\mathrm{rel}} u_{R} \frac{\mathrm{d} F}{\mathrm{~d}\left(\gamma R_{\mathrm{rel}}\right)} .
$$

\section{COMPARISON With TIME-DOMAIN SPECTROSCOPY}

Having established the linearity of the KM remission function with respect to concentration for each of the four frequencies used in this work, a direct comparison was made between the DR results and those obtained using $\mathrm{THz}$ time-domain spectroscopy (TDS). For these measurements, samples were pressed mechanically into circular pellets with diameter $8 \mathrm{~mm}$ and a thickness of $0.3-0.4 \mathrm{~mm}$. The experimental method has been described in detail previously [17], and is summarized as follows. THz radiation was generated from a low-temperature-grown GaAs photoconductive switch using $\sim 10$ fs pulses from a near-infrared (NIR) Ti:sapphire laser as the optical pump. The emitted broadband (pulsed) $\mathrm{THz}$ radiation was focused onto the sample using a pair of off-axis parabolic mirrors, and the transmitted fraction of the radiation was measured using a time-resolved electro-optic sampling approach, which was triggered using a fraction of the NIR radiation from the Ti:sapphire laser (coherent with the excitation pulse). The time-variation of the $\mathrm{THz}$ field was sampled by using an opto-mechanical delay stage to vary the time interval between the excitation pulse and the probe pulse. The spectrum 
of the transmitted THz pulse was obtained from a Fourier transform of the time-domain samples, and compared with a reference spectrum (obtained with no sample in the pulse-propagation path) to obtain the absorption spectrum of the pellet.

Fig. 5 shows the THz TDS spectra (solid lines) of three pellets of cellulose, with mass-concentrations of $5 \%, 15 \%$, and $100 \%$. In all cases, the measured absorption was confirmed to be within the dynamic range of the measurement scheme, as described in [18]. In (2), the factor $\alpha(c)=S F\left(\gamma R_{\mathrm{rel}}\right) / \beta$, should strictly be taken as a frequency-dependent function. However, the measured median particle sizes for all powders considered in this work lie in the $\sim 10-50 \mu \mathrm{m}$ range, which is comparable to the wavelength of the THz radiation within the materials of interest. As such, the scattering behaviour is outside the Rayleigh regime, and its dependence on wavelength can be assumed to be weak over the relatively small range of frequencies considered here, compared with the frequency dependence of the absorption coefficient. Furthermore, over this frequency range, the refractive index of PTFE is invariant and therefore, $S / \beta$ can be taken as a global constant for a given sample-PTFE admixture, regardless of concentration or frequency. Under these approximations, a single approximately linear relationship exists between the BL absorption coefficient and the KM remission function, for the entire range of concentrations and frequencies studied.

The measured TDS absorption coefficient $\alpha_{\text {TDS }}$ was plotted against the equivalent $F\left(\gamma R_{\text {rel }}\right)$ values from Fig. 4(b) for the three cellulose concentrations at all four frequencies. The expected linear relationship was recovered, with a scaling factor of $S / \beta=5.23 \pm 0.17 \mathrm{~mm}^{-1}$. The symbols in Fig. 5 show the absorption coefficient calculated from the KM model, plotted on the same axes as the directly-measured TDS absorption coefficient. The magnitude of the absorption coefficient is correct in all instances, although the frequency-dependence is reproduced most accurately at $15 \%$ concentration. The standard uncertainty in the inferred absorption coefficeient is also shown to be similar to that of the TDS measurement. The deviation of the the KM model from the TDS absorption at very high and very low concentrations is well known, however [15], since the weak absorption coefficient of the matrix material becomes significant at low concentrations, and the concentration-dependence of the scattering coefficient becomes significant at high concentrations. Although the absolute value of the absorption coefficient can only be recovered a posteriori by using the fitting parameter, $S / \beta$, the shape of the unscaled spectrum can still be used for spectroscopic analysis of powders, including spectral lineshapes and relative absorption at different frequencies. Indeed, the scaling is unnecessary for common automated spectral recognition techniques such as principal component analysis [19], since the scaling of eigenfunctions is arbitrary within such methods. Hence, $\gamma$ is the only fitting parameter required in this type of analysis. Here, we hypothesize that since $\gamma$ is predominantly a measure of the reflectance of the reference material, a single value $\gamma=1.03$ (as determined above) may be used for the analysis of a wide range of materials, provided that the angular divergence of the scattered radiation is similar. Having obtained this 'universal' estimate of $\gamma$ from samples of cellulose, it is now possible to analyze individual samples of additional materials directly without the need to study multiple samples with different concentrations.

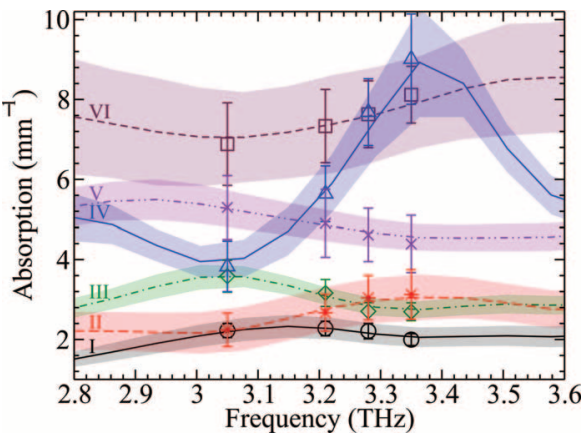

Fig. 6. TDS absorption coefficients (solid lines) for a range of powdered materials, all diluted to a concentration of $15 \%$ within an admixture with PTFE. The scaled KM remission functions obtained from DR measurements are plotted on the same axes as open symbols. Powdered materials are: (I, circles) caffeine, (II, stars) sucrose, (III, diamonds) cellulose, (IV, triangles) granulated sugar, (V, crosses) lydocaine, (VI, squares) benzocaine. The standard uncertainty in absorption coefficient is represented as semi-transparent bands for the TDS data, and as error bars for the DR data.

TABLE II

SCALING FACTOR $S / \beta\left(\mathrm{mm}^{-1}\right)$ FOR KM REMISSION FUNCTION ANALYSIS OF A RANGe OF Materials WITH 15\% CONCENTRATION IN ADMIXTURE WITH PTFE

\begin{tabular}{l|r}
\hline Material & Scaling factor \\
\hline \hline Benzocaine & $27.2 \pm 0.3$ \\
Caffeine & $4.04 \pm 0.05$ \\
Cellulose & $5.68 \pm 0.09$ \\
Granulated sugar & $35.7 \pm 0.6$ \\
Lydocaine & $19.9 \pm 0.3$ \\
Sucrose & $26.4 \pm 0.5$ \\
\hline
\end{tabular}

A range of powdered samples was prepared, using a single concentration of $15 \%$ in each case. The TDS absorption spectra were measured and are plotted in Fig. 6 for each sample material. It can be seen that all the materials studied have BL absorption coefficients in the range $2-10 \mathrm{~mm}^{-1}$. DR measurements were undertaken for each of the powdered samples, and the $\mathrm{KM}$ remission function was calculated, using the fixed, estimated value of $\gamma=1.03$ as above. The scaling factor $S / \beta$ was determined for each sample, using linear regression to the TDS absorption coefficients at each frequency, as was the case for the cellulose samples above (albeit at a single concentration). The results of this regression analysis are presented in Table II. It is shown that in all cases, the linear fit to the TDS model is robust (standard uncertainty within $1 \%-2 \%$ ) and the scaling factor varies from $\sim 4-40 \mathrm{~mm}^{-1}$. Since the concentration of the absorbing powders is low in these samples, the refractive index can be assumed to be close to that of PTFE in all cases. As such, we attribute the variation in $S / \beta$ principally to the difference in scattering coefficient between the materials. The scaled absorption coefficient for each material (from the KM model) is plotted on the same axes as the TDS absorption coefficient in Fig. 6, showing that in all cases, the shape of the spectral features is resolved with high accuracy.

\section{CONCLUSION}

We have demonstrated a frequency-switchable diffuse reflectance $\mathrm{THz}$ spectroscopy system, based on a THz QCL operating at $3.06,3.21,3.28$, and $3.35 \mathrm{THz}$ and have used this 
system to resolve spectral features in powdered media. We have shown that a simple KM model can be used to recover key properties of the $\mathrm{THz}$ absorption spectra, in good agreement with results obtained using conventional $\mathrm{THz}$ time-domain spectroscopy techniques. The KM remission function was shown to be proportional to the BL absorption coefficient, enabling direct determination of absorption features from DR measurements without the need for a priori knowledge of the refractive index or scattering properties of the powders. The KM absorption was shown to scale reliably with respect to the concentration of the absorbing powder over a wide range of values, and was found to reproduce the shape of TDS spectra accurately for a wide range of materials (including pharmaceutical compounds, foodstuffs, and chemical precursors) for powder concentrations of $15 \%$ in admixture with PTFE. Although the absolute value of the BL absorption coefficient is not retrieved directly by this technique, we have shown that absolute values can be obtained through the use of an experimentally-determined, material-dependent scaling factor, and this scaling factor is constant over the $300 \mathrm{GHz}$ bandwidth studied in this work. As such, the technique could potentially be applied directly (without scaling) to automated real-time material identification techniques such as principal component analysis within pharmaceutical studies or security screening.

In our analysis, the scattering coefficient was assumed to be frequency-independent. However, in principle, a more accurate frequency-dependent scattering model could be derived for a known particle distribution. Furthermore, the absolute value of the absorption coefficient could, in principle, be obtained directly through the use of more sophisticated analysis (e.g., quasi-crystalline approximations). It is important to note that the speed of the DR spectroscopy technique is determined principally by the mechanical translation stage and could be improved significantly using fast scanning optics. Additionally, through precise optical alignment, the spatial-resolution of the present system could be optimised for imaging applications. Finally, although we have employed a laser that was tunable over four discrete emission lines in the present work, the technique is equally applicable to alternative $\mathrm{THz}$ QCL tuning approaches such as those based on discrete Vernier tuning [20], external cavities [21] or aperiodic gratings [22].

\section{ACKNOWLEDGMENT}

The authors are grateful to K. Tych, University of Leeds, U.K., for assistance with optical microscopy of samples; and to T. Al-Abdullah and S. Nie, University of Leeds, U.K., for assistance with data acquisition.

\section{REFERENCES}

[1] J. A. Zeitler, P. F. Taday, D. A. Newnham, M. Pepper, K. C. Gordon, and T. Rades, "Terahertz pulsed spectroscopy and imaging in the pharmaceutical setting - A review," J. Pharm. Pharmacol., vol. 59, no. 2, pp. 209-223, 2007.

[2] A. J. Fitzgerald, E. Berry, N. N. Zinovev, G. C. Walker, M. A. Smith, and J. M. Chamberlain, "An introduction to medical imaging with coherent terahertz frequency radiation," Phys. Med. Biol., vol. 47, no. 7, Apr. 2002, Art no R67.

[3] K. Kawase, Y. Ogawa, Y. Watanabe, and H. Inoue, "Non-destructive terahertz imaging of illicit drugs using spectral fingerprints," Opt. Express, vol. 11, no. 20, pp. 2549-2554, Oct. 2003.
[4] A. G. Davies, A. D. Burnett, W. Fan, E. H. Linfield, and J. E. Cunningham, "Terahertz spectroscopy of explosives and drugs," Mater. Today, vol. 11, no. 3, pp. 18-26, 2008.

[5] H.-B. Liu, Y. Chen, G. J. Bastiaans, and X.-C. Zhang, "Detection and identification of explosive RDX by $\mathrm{THz}$ diffuse reflection spectroscopy," Opt. Express, vol. 14, no. 1, pp. 415-423, Jan. 2006.

[6] D. J. J. Fraser and P. R. Griffiths, "Effect of scattering coefficient on diffuse reflectance infrared spectra," Appl. Spectrosc., vol. 44, no. 2, pp. 193-199, Feb. 1990.

[7] P. Dean et al., "Absorption-sensitive diffuse reflection imaging of concealed powders using a terahertz quantum cascade laser," Opt. Express, vol. 16, no. 9, pp. 5997-6007, Apr. 2008.

[8] P. Dean et al., "Measurement and analysis of the diffuse reflectance of powdered samples at terahertz frequencies using a quantum cascade laser," J. Chem. Phys., vol. 134, no. 13, p. 134304, Apr. 2011.

[9] S. P. Khanna, M. Salih, P. Dean, A. G. Davies, and E. H. Linfield, "Electrically tunable terahertz quantum-cascade laser with a heterogeneous active region," Appl. Phys. Lett., vol. 95, no. 18, p. 181101, Nov. 2009.

[10] H. Luo, S. R. Laframboise, Z. R. Wasilewski, G. C. Aers, H. C. Liu and J. C. Cao, "Terahertz quantum-cascade lasers based on a three-well active module," Appl. Phys. Lett., vol. 90, no. 4, Jan. 2007, Art no 041112.

[11] S. P. Khanna, S. Chakraborty, M. Lachab, N. M. Hinchcliffe, E. H. Linfield, and A. G. Davies, "The growth and measurement of terahertz quantum cascade lasers," Physica E, vol. 40, no. 6, pp. 1859-1861, Apr. 2008.

[12] J. W. Coltman, "The specification of imaging properties by response to a sine wave input," J. Opt. Soc. Amer., vol. 44, no. 6, p. 468, 1954.

[13] H. Rinne, The Weibull Distribution: A Handbook. Boca Raton, FL, USA: CRC Press, 2008.

[14] L. Tsang and J. A. Kong, "Multiple scattering of electromagnetic waves by random distributions of discrete scatterers with coherent potential and quantum mechanical formalism," J. Appl. Phys., vol. 51, no. 7, pp. 3465-3485, Jul. 1980.

[15] J. D. Lindberg, "Absolute diffuse reflectance from relative reflectance measurements," Appl. Opt., vol. 26, no. 14, pp. 2900-2905, Jul. 1987.

[16] J. D. Lindberg, R. E. Douglass, and D. M. Garvey, "Absorption-coefficient-determination method for particulate materials," Appl. Opt., vol. 33, no. 19, pp. 4314-4319, Jul. 1994.

[17] A. D. Burnett et al., "Calculation and measurement of terahertz active normal modes in crystalline PETN," Chem. Phys. Chem., vol. 11, no. 2, pp. 368-378, 2010.

[18] P. U. Jepsen and B. Fischer, "Dynamic range in terahertz time-domain transmission and reflection spectroscopy," Opt. Lett., vol. 30, no. 1, pp. 29-31, Jan. 2005.

[19] A. D. Burnett et al., "Broadband terahertz time-domain spectroscopy of drugs-of-abuse and the use of principal component analysis," Analyst, vol. 134, no. 8, pp. 1658-1668, Jul. 2009.

[20] I. Kundu et al., "Discrete Vernier tuning in terahertz quantum cascade lasers using coupled cavities," Opt. Express, vol. 22, no. 13, pp. 16595-16605, Jun. 2014.

[21] M. Vitiello and A. Tredicucci, "Tunable emission in THz quantum cascade lasers," IEEE Trans. THz Sci. Technol., vol. 1, no. 1, pp. 76-84, Sep. 2011.

[22] S. Chakraborty, O. Marshall, C. W. Hsin, M. Khairuzzaman, H. Beere, and D. Ritchie, "Discrete mode tuning in terahertz quantum cascade lasers," Opt. Express, vol. 20, no. 26, Dec. 2012, Art no B306.

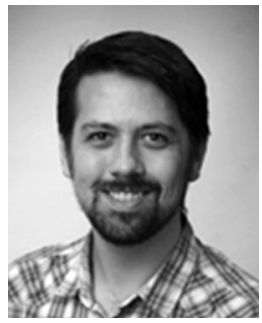

Alex Valavanis received the M.Eng. (Hons) degree in electronic engineering from the University of York, York, U.K., in 2004, and the Ph.D. degree in electronic and electrical engineering from the University of Leeds, Leeds, U.K., in 2009.

From 2004 to 2005, he was an instrumentation engineer with STFC Daresbury Laboratories, Warrington, U.K. He is currently a Research Fellow at the University of Leeds, Leeds, U.K. His research interests include quantum cascade lasers, terahertz sensors, silicon photonics, and computational methods for quantum electronics.

Dr. Valavanis is a member of the Institution of Engineering and Technology (IET), U.K. 


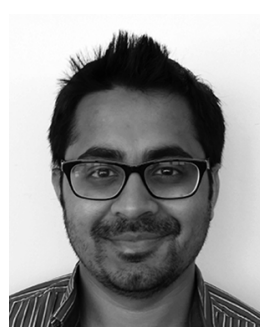

Siddhant Chowdhury was born in Kolkata, India, in 1990. He received the B.Eng. degree in electronics and nanotechnology from the University of Leeds, Leeds, U.K., in 2011, where he is currently working towards the Ph.D. degree.

Following graduation, he was a Summer Intern with the Institute of Microwaves and Photonics, University of Leeds, Leeds, U.K. His research interests include terahertz quantum cascade lasers, photomixers and their applications.

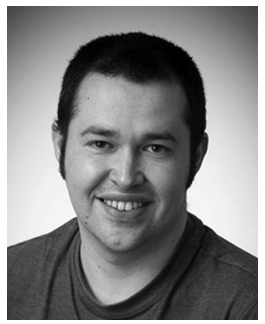

Andrew D. Burnett received the M.Chem. degree in Chemistry from the University of Bradford, Bradford, U.K., in 2004, and the Ph.D. degree in terahertz spectroscopy at the University of Leeds, Leeds, U.K., in 2008 .

Since then, he has continued his work at at the University of Leeds, Leeds, U.K., and was awarded a Postdoctoral Fellowship in 2011 by the Engineering and Physical Sciences Research Council, U.K. He is currently a teaching and research fellow in Physical Chemistry and THz Technology.

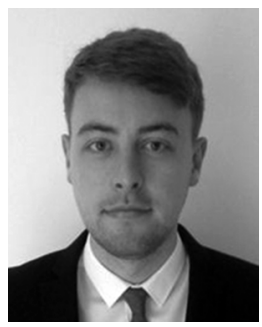

Adam R. Clarkson received the M.Eng. (Hons) degree in electronic and electrical engineering from the University of Leeds, Leeds, U.K., in 2014

$\mathrm{He}$ is currently an Implementation Engineer with ARM, Sheffield, U.K.

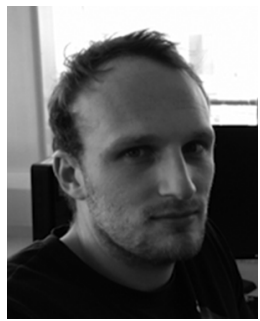

David R. Bacon received the M.Eng. degree in electronic and electrical engineering from the University of Leeds, Leeds, U.K. in 2013, where he is currently working towards the Ph.D. degree.

His research interests include terahertz quantum cascade lasers, time-domain spectroscopy and ultrafast electro-optics.

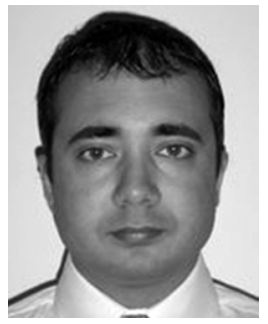

Suraj P. Khanna received the B.Eng. degree in electronics and telecommunications engineering from the University of Amravati, Amravati, India, in 2001, and the M.Sc. degree in nanoscale science and technology, and the Ph.D. degree in electronic and electrical engineering from the University of Leeds, Leeds, U.K., in 2004 and 2008, respectively.

From 2008 to 2011, he was a Postdoctoral Research Associate at the University of Leeds and from 2011 to 2012, he was a Research Fellow at Northwestern University, USA. He is currently Principal Research Scientist with the National Physical Laboratory, New Delhi, India.
His research interests include molecular beam epitaxial growth, MOCVD growth of dilute magnetic semiconductors and nitride-based materials, semiconductor device fabrication, quantum cascade lasers, and terahertz-frequency optical systems.

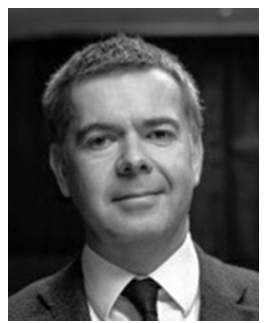

Alexander Giles Davies received the B.Sc. (Hons.) degree in Chemical Physics from the University of Bristol, U.K., in 1987, and the Ph.D. degree in semiconductor physics from the University of Cambridge, U.K., in 1991.

In 1991, he joined the University of New South Wales, Sydney, Australia, supported by an Australian Research Council Fellowship, before returning to the Cavendish Laboratory, University of Cambridge, U.K., in 1995 as a Royal Society University Research Fellow, and subsequently Trevelyan Fellow of Selwyn College, Cambridge University. Since 2002, he has been with the School of Electronic and Electrical Engineering, University of Leeds, Leeds, U.K., as Professor of Electronic and Photonic Engineering, and is currently also Pro-Dean for Research and Innovation in the Faculty of Engineering. His research interests include the optical and electronic properties of semiconductor devices, terahertz frequency electronics and photonics, and the exploitation of biological properties for nanostructure engineering.

Professor Davies is a Fellow of the Institute of Physics, and both a Chartered Physicist and Engineer in the U.K. He received a Wolfson Research Merit award from the Royal Society in 2011, and shared the Faraday Medal and Prize from the Institute of Physics in 2014.

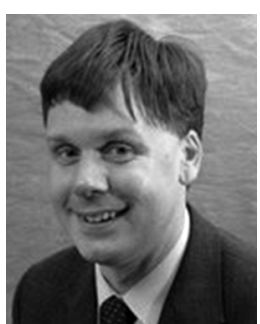

Edmund H. Linfield received the B.A. (Hons.) degree in physics and the M.A. degree from the University of Cambridge, Cambridge, U.K., in 1986 and 1991, respectively, and the $\mathrm{Ph} . \mathrm{D}$. degree in semiconductor physics from the Cavendish Laboratory, University of Cambridge, in 1991.

He continued his research as a Post-Doctoral Researcher Associate with the Cavendish Laboratory, University of Cambridge, U.K., until 1997, when he was appointed as an Assistant Director of Research. $\mathrm{He}$ also became a Fellow of Gonville and Caius College, Cambridge. In 2004, he moved to the University of Leeds, Leeds, U.K., to take up the Chair of Terahertz Electronics. He is currently also Director of Research in the School of Electronic and Electrical Engineering, and was awarded a "Dream Fellowship" from the Engineering and Physical Sciences Research Council (2011). His research interests include semiconductor growth and device fabrication, terahertz-frequency optics and electronics, and nanotechnology.

Prof. Linfield received the IOP Faraday Medal and Prize, jointly with Prof. Davies, in 2014 and a Wolfson Research Merit award from the Royal Society in 2015 .

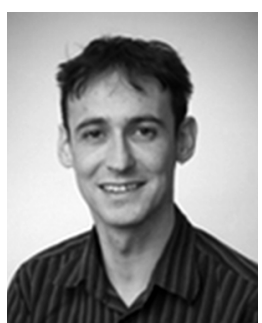

Paul Dean received the M.Phys. (Hons.) degree in physics in 2001 and the Ph.D. degree in laser physics in 2005, both from the University of Manchester, Manchester, U.K.

In 2005, he was appointed as a Post-Doctoral Research Associate at the Institute of Microwaves and Photonics, School of Electronic and Electrical Engineering, University of Leeds, Leeds, U.K., and as a University Research Fellow in 2011. His research interests include terahertz optoelectronics, quantum cascade lasers, and terahertz imaging techniques. 